\title{
CORRECTION
}

\section{Correction to: Implicit facial emotion recognition of fear and anger in obesity}

\author{
Federica Scarpina ${ }^{1,2}$ (D) Giorgia Varallo ${ }^{3,4}$. Gianluca Castelnuovo ${ }^{3,4} \cdot$ Paolo Capodaglio $^{5}$. Enrico Molinari ${ }^{3,4}$. \\ Alessandro Mauro $^{1,2}$
}

Published online: 8 October 2020

(c) Springer Nature Switzerland AG 2020

\section{Correction to: \\ Eating and Weight Disorders - Studies on Anorexia, Bulimia and Obesity https://doi.org/10.1007/s40519-020-01010-6}

Unfortunately, the last author name was incorrectly published in the original publication. The complete correct name is given below.

Alessandro Mauro

The original article has been updated.
Publisher's Note Springer Nature remains neutral with regard to jurisdictional claims in published maps and institutional affiliations.

The original article can be found online at https://doi.org/10.1007/ s40519-020-01010-6.

Federica Scarpina

f.scarpina@auxologico.it; federica.scarpina@unito.it

1 Istituto Auxologico Italiano, IRCCS, U.O. di Neurologia

e Neuroriabilitazione, Ospedale San Giuseppe,

28824 Piancavallo, VCO, Italy

2 "Rita Levi Montalcini”, Department of Neurosciences, University of Turin, 10124 Turin, Italy

3 Istituto Auxologico Italiano, IRCCS, Laboratorio di Psicologia, Ospedale San Giuseppe, 28824 Piancavallo, Italy

4 Psychology Department, Università Cattolica del Sacro Cuore, 20123 Milan, Italy

5 Istituto Auxologico Italiano, IRCCS, U.O. di. Riabilitazione Osteoarticolare, Ospedale S. Giuseppe, 28824 Piancavallo, Italy 